\title{
Enfermedad celíaca y desarrollo de patologías secundarias
}

\section{Celiac disease and the development of secondary disorders}

\author{
Daniel Solano-Sánchez¹, Daniel A. Quesada-Yamasaki²
}

\begin{abstract}
Resumen. La enfermedad celíaca es una patología autoinmune sistémica caracterizada por un estado inflamatorio crónico de la mucosa y submucosa intestinal, de origen multifactorial y detonada por factores ambientales como la ingesta de gluten en individuos genéticamente predispuestos a sensibilizarse a dicha proteína. La prevalencia de la enfermedad a nivel mundial se estima entre 1,1\% y 1,7\%, según datos de seroprevalencia. Sin embargo, se considera que existe una importante cantidad de individuos no diagnosticados, quienes podrían llegar a desarrollar una serie de complicaciones debido a que no reciben el manejo adecuado. Por ello, el objetivo de esta revisión comprende valorar algunas de las patologías secundarias más frecuentes asociadas a la enfermedad celíaca, evidenciando la afectación sistémica que puede presentar un individuo con esta enfermedad.
\end{abstract}

Palabras clave: enfermedad celíaca, enfermedades autoinmunes, predisposición genética a la enfermedad, prueba de laboratorio.

Abstract. Celiac disease is a systemic autoimmune pathology characterized by a chronic inflammatory state of the intestinal mucosa and submucosa, of multifactorial origin and triggered by environmental factors such as gluten intake in individuals genetically predisposed to become sensitized to such protein. The worldwide prevalence of the disease is estimated to be between $1.1 \%$ and $1.7 \%$, based on seroprevalence data. However, it is considered that there are a significant number of undiagnosed individuals, who could develop a series of complications due to not receiving adequate management. For this reason, the objective of this review is to assess some of the most frequent secondary disorders associated with celiac disease, evidencing the systemic involvement that an individual with this disease can develop.

\footnotetext{
${ }^{1}$ Microbiólogo y Químico Clínico. Caja Costarricense de Seguro Social. Heredia, Costa Rica. E-mail: danielsolano934@gmail.com.

${ }^{2}$ Microbiólogo y Químico Clínico. Regente de Laboratorio, Laboratorios Cartín. Alajuela, Costa Rica.

Conflicto de interés: los autores declaran que no tienen conflicto de interés.

Medicina \& Laboratorio 2020;24:291-305. https://doi.org/10.36384/01232576.337

Recibido el 12 de mayo de 2020; aceptado el 30 de julio de 2020. Editora Médica Colombiana S.A., $2020^{\circ}$.
} 
Keywords: celiac disease, autoimmune diseases, genetic predisposition to disease, laboratory test.

\section{Introducción}

La enfermedad celíaca (EC), según la undécima revisión de la Clasificación Internacional de Enfermedades (ClE11), es una intolerancia permanente a las proteínas del gluten, de origen autoinmunitario y caracterizada por un estado inflamatorio crónico de la mucosa y la submucosa intestinales, pudiendo causar alteraciones en la digestión y la absorción de nutrientes [1].

También conocida como esprúe tropical, la EC es considerada una enfermedad multifactorial. Esto porque, aunque puede ser detonada por factores ambientales como la ingesta de gluten, existe consenso sobre su presentación con mayor frecuencia en individuos con predisposición genética [2]. La alta heredabilidad es de carácter poligénico, con un patrón de herencia no mendeliano e involucra tanto haplotipos del complejo de antígenos leucocitarios humanos (HLA, del inglés, Human Leukocyte Antigens), principalmente HLA-DQ2 y HLA-DQ8, así como polimorfismos de un solo nucleótido (SNPs, del inglés, Single-Nucleotide Polymorphisms) en otros loci que, conjuntamente, proporcionan un riesgo aumentado de desarrollar la enfermedad $[2,3]$.

En términos generales respecto a la fisiopatología (ver figura 1), una vez que la gliadina (proteína contenida en el gluten) es liberada en el intestino, ocurre su interacción con las células intestinales, pudiendo inducir desensamblaje de uniones estrechas entre los enterocitos, que culmina con la expresión de zonu- lina. La zonulina activada aumenta la permeabilidad intestinal, permitiendo que los péptidos de la gliadina pasen a través de la barrera epitelial, activando linfocitos T CD4+ de la lámina propia, que, a su vez, estimulan la producción de citoquinas proinflamatorias. Toda esta cadena de eventos culmina con la activación de las respuestas Th1 y Th2, además de una liberación de interferón gamma (IFN- $\gamma$ ) y expansión clonal de los linfocitos $B$, la diferenciación de células plasmáticas y la producción de anticuerpos anti-gliadina y anti-transglutaminasa, principales marcadores de laboratorio empleados en el diagnóstico de la patología [4].

La prevalencia de la enfermedad a nivel mundial se estima entre 1,1\% y $1,7 \%$, según datos de seroprevalencia, es decir, confirmación diagnóstica por la presencia de anticuerpos anti-transglutaminasa tisular o anticuerpos antiendomisio en el suero de pacientes [5]. En cambio, si la estimación se realiza con base en resultados de biopsias positivas para EC, la prevalencia global es un poco menor, calculada en un $0,7 \%$ [5]. Estas estimaciones de prevalencia presentan variaciones por región geográfica (mayor prevalencia en Europa y Oceanía), sexo (mayor en mujeres) y edad (mayor en niños) [5].

Aunque el diagnóstico de EC se ha optimizado con el tiempo [6], continúa existiendo un importante porcentaje de pacientes que no son diagnosticados o que reciben un diagnóstico tardío, con estimaciones que afirman, incluso, que por cada paciente diagnosticado, siete personas con EC aún no han recibido 


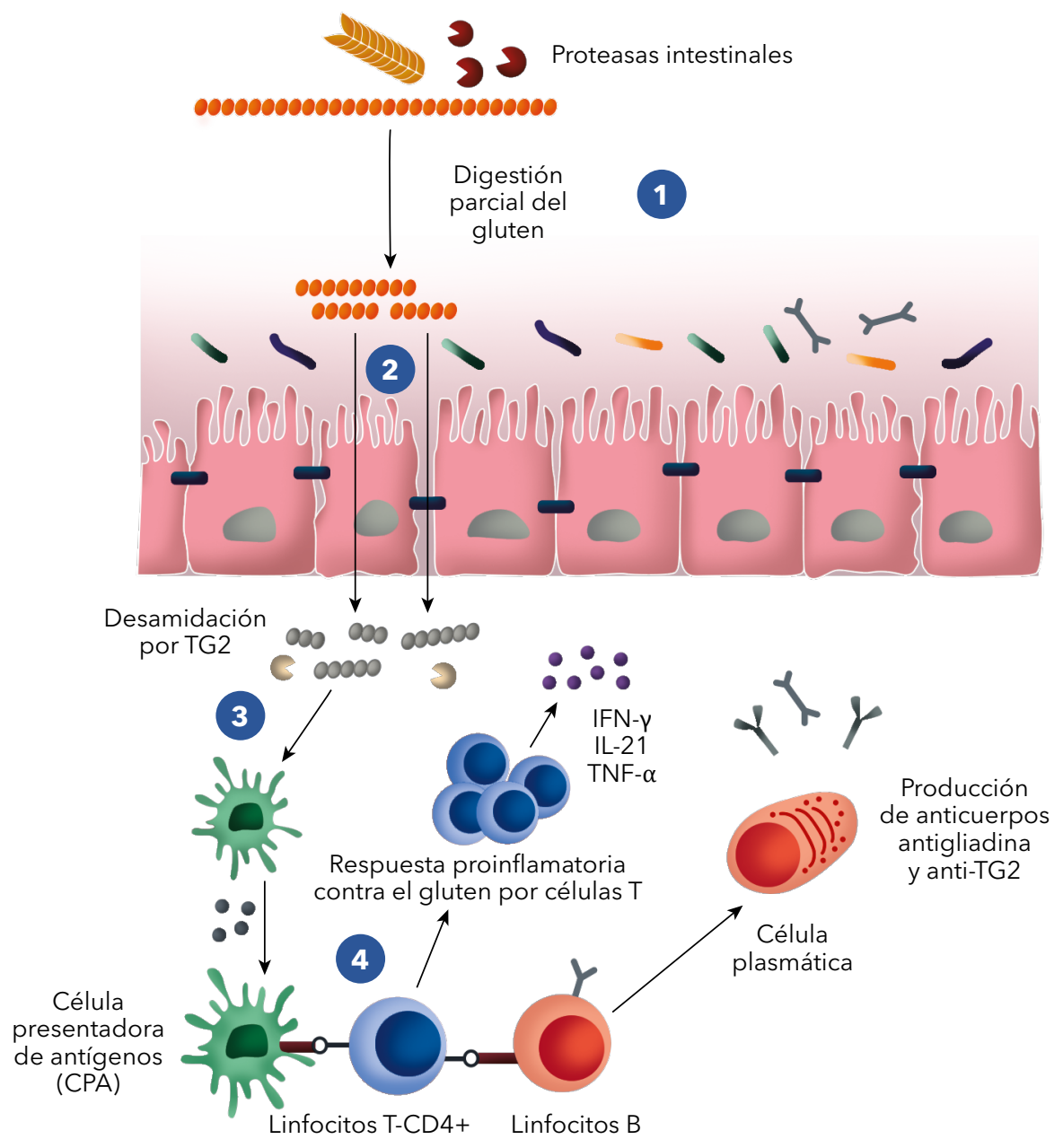

Figura 1. Fisiopatología de la enfermedad celíaca. (1) La digestión parcial del gluten permite la interacción de la gliadina con el epitelio intestinal, lo cual puede inducir (2) el desensamblaje de las uniones estrechas entre enterocitos, y la activación de la zonulina con un aumento de la permeabilidad intestinal. (3) Esto conduce a que los péptidos de gliadina atraviesen la barrera intestinal y, en capas inferiores de la mucosa, sufran desamidación por la transglutaminasa tisular (tTG o TG2), lo cual facilita su presentación por células presentadoras de antígenos (CPA) portadoras de las moléculas de HLA-DQ relevantes para la enfermedad. (4) A partir de allí, se desencadena una respuesta inmunológica tanto celular como humoral dirigida contra epítopes de la gliadina y de la TG2, la cual explica en gran parte la sintomatología con la que cursa el paciente con enfermedad celíaca. IFN- $\gamma$ : interferón gamma; TNF- $\alpha$ : factor de necrosis tumoral alfa; IL-21: interleuquina 21.

diagnóstico $[7,8]$. Si bien la EC puede ser manejada adecuadamente sin afecciones significativas a la calidad de vida del paciente cuando se diagnostica de manera temprana, un diagnóstico tar- dío puede llevar a la exacerbación de las manifestaciones clínicas y representar un mayor riesgo de desarrollo de complicaciones o enfermedades secundarias [9]. 
La combinación de la sintomatología de la EC, junto con las consecuencias de las enfermedades secundarias, pueden conllevar una afectación significativa en la calidad de vida de los pacientes e incluso desencadenar condiciones que implican un riesgo grave. Al respecto, un análisis reciente de las tendencias en investigación sobre las manifestaciones extra-intestinales de la EC, mostró una disminución en las investigaciones sobre complicaciones óseas y nerviosas, lo que resulta preocupante ya que podría implicar una desaceleración en el progreso sobre la comprensión de estos aspectos de la EC [10].

Por todo ello, a través de la presente investigación se persigue el objetivo de revisar los aspectos más relevantes (fisiopatología, manifestaciones clínicas y apoyos diagnósticos del laboratorio) de las principales patologías o condiciones que se desarrollan de manera secundaria a la enfermedad celíaca.

\section{Patologías secundarias a enfermedad celíaca}

En este punto conviene aclarar la distinción entre enfermedades o condiciones secundarias a la EC y patologías asociadas. Una enfermedad secundaria es aquella que es una complicación y/o que ocurre como resultado de la condición primaria (en este caso, EC). Por su parte, las patologías asociadas son aquellas que existen simultáneamente con la EC y podrían o no tener factores subyacentes comunes, pero no son consecuencia directa de la EC [11].

En el contexto de la EC, los problemas de malabsorción, una condición subyacente de autoinmunidad y una mayor propensión a un estado general proinflamatorio, hacen más vulnerables a los pacientes a padecimientos secundarios. Entre las complicaciones - manifestaciones extraintestinales más comunes de la EC se encuentran: desbalances óseos como osteopenia y osteoporosis, daño hepático, anemias, dermatitis herpetiforme y manifestaciones neurológicas, por ejemplo, neuropatías y cefaleas, entre otras [12]. A lo largo de los siguientes apartados se abordará con más detalle cada una de ellas.

\section{Osteopenia y osteoporosis}

Por definición, la osteoporosis comprende una condición clínica con pérdida y deterioro progresivo de la masa ósea y su microarquitectura, lo que aumenta la fragilidad del hueso y el riesgo de presentar fracturas [13]. La osteopenia, en cambio, se refiere a la condición en la cual la densidad ósea es más baja de lo normal, pero aún no ha alcanzado los niveles bajos observados en la osteoporosis [13].

Los pacientes con enfermedad celíaca pueden presentar una masa ósea disminuida por la reducción en la ingesta de calcio y su absorción intestinal; incluso se ha documentado baja ingesta de proteínas que conduce a una disminución en la síntesis de factor de crecimiento insulínico (IGF-1) por parte del hígado, necesario para el adecuado crecimiento, proliferación y diferenciación de los condrocitos en el cartílago, los osteoblastos y el estímulo de la síntesis de colágeno [14].

Se ha descrito otro mecanismo relacionado con la autoinmunidad. Al ser una enfermedad autoinmune sistémica, los pacientes con EC presentan anticuerpos IgA contra estructuras intra y extracelulares del tejido óseo. El daño se da cuando 
estos anticuerpos reconocen la transglutaminasa del tejido óseo como un antígeno y forman inmunocomplejos [14].

La mala absorción y el desarrollo de un estado proinflamatorio crónico influyen en la disminución de la densidad mineral ósea, por lo que se recomienda que pacientes con enfermedad celíaca tengan un monitoreo constante de los niveles de calcio, paratohormona (PTH) y 25-OH-vitamina D. Además, el tratamiento para el paciente debe hacerse durante un año tras haber iniciado una dieta libre de gluten (DLG) y no difiere del de otras osteoporosis [15].

\section{Daño hepático}

Los mecanismos de daño hepático en pacientes con EC son poco conocidos; sin embargo, elevaciones de las transaminasas son hallazgos frecuentes en pacientes con enfermedad celíaca no tratada [16]. Como principal hipótesis de la patogénesis se destaca el aumento en la permeabilidad intestinal, lo que favorecería la absorción de antígenos procedentes del intestino por medio de la circulación portal. Este incremento de la permeabilidad a los antígenos intraluminales puede generar una respuesta inmunológica en los individuos genéticamente predispuestos, contra antígenos que comparten epítopes propios de las proteínas hepáticas o contra antígenos de la mucosa intestinal, inducidos por la reacción hacia la gliadina. En la mayoría de pacientes se observa una normalización de las transaminasas tras el primer año de DLG [17]; no obstante, algunos pacientes tras un año con DLG mantienen las transaminasas elevadas y pueden cursar con esteatosis hepática [18].

Es necesario recordar la posibilidad de pacientes que tengan resultados falsos positivos para anticuerpos anti-transglutaminasa tisular (anti-tTG) tipo IgA o lgG, y que cursen con enfermedad hepática crónica por diferentes etiologías [18].

Existe otra teoría respecto al daño hepático donde la respuesta inmunológica podría estar producida por neoantígenos, que se creen pueden ser generados por un sobrecrecimiento bacteriano, secundario a un tránsito intestinal prolongado, o bien, inducidos por la transformación de antígenos de la dieta mediante la acción de la transglutaminasa [19].

Además, hay una asociación entre la EC y la hepatitis crónica autoinmune, principalmente porque en ambas patologías se comparten combinaciones de genes que codifican los antígenos HLA de clase II [18]. Se estima que aproximadamente un $20 \%$ de pacientes con hepatitis autoinmune presentan atrofia vellositaria intestinal; sin embargo, la prevalencia de pacientes con EC que manifiestan hepatitis autoinmune es inferior al 1\% [19].

\section{Anemia}

La anemia microcítica hipocrómica es una de las manifestaciones clínicas no gastrointestinales más frecuentes de la enfermedad celíaca. El déficit de hierro en pacientes con EC viene dado principalmente por la mala absorción intestinal inducida por la atrofia vellositaria. Aproximadamente un 50\% de los adultos no presentan síntomas clásicos de EC; no obstante, cursan con anemia ferropénica [20].

La anemia por déficit de hierro es de las principales manifestaciones de una forma subclínica de enfermedad celíaca; es por esto que se recomienda descartar EC en cualquier individuo que curse con anemia ferropénica de ori- 
gen incierto o refractaria al tratamiento con hierro oral [21].

Adicionalmente, las alteraciones en la absorción de otros compuestos pueden conducir a otros tipos de anemias por deficiencias nutricionales, como la anemia megaloblástica, producto de niveles insuficientes de vitamina B12 [22] o ácido fólico [23]. Al mismo tiempo, el estado proinflamatorio crónico y la liberación de citoquinas proinflamatorias que puede presentarse de manera continua en la EC, pueden llevar al desarrollo de una anemia por inflamación (o anemia de la enfermedad crónica) [23]. Debido a que los problemas inflamatorios y absortivos pueden presentarse simultáneamente en la EC, no es de extrañar que una proporción importante de pacientes presente anemias de etiología multifactorial [23]. Por ello, es recomendable llevar a cabo un abordaje integral en el hallazgo de la(s) causa(s) y manejo de los pacientes con esta manifestación hematológica secundaria a la EC.

\section{Dermatitis herpetiforme}

La dermatitis herpetiforme comprende la principal manifestación cutánea de la intolerancia al gluten y clínicamente se manifiesta como una enfermedad ampollar autoinmune con erupción papulovesicular pruriginosa de extensión en superficies de las extremidades [24,25].

Desde el punto de vista de la patogenia, existe una estrecha relación genética entre ambas enfermedades con los haplotipos HLA-DQ2 y HLA-DQ8, concretamente con los haplotipos DQA1*0501 y DQB*0201 localizados en el cromosoma $6[24,25]$.

Como bien se conoce, la transglutaminasa tisular (tTG) es el autoantíge- no más importante en la enfermedad celíaca, mientras que en la dermatitis herpetiforme el autoantígeno parece ser la transglutaminasa epidérmica (eTG), responsable del mantenimiento de la envoltura celular cornificada en la epidermis. Conviene aclarar que la relación inmunológica entre ambas enfermedades no son los autoantígenos, pues difieren entre ellas, sino más bien la presencia de epítopes compartidos $[24,25]$.

Los autoanticuerpos circulantes en la EC son lgA e lgG dirigidos contra la tTG y la gliadina, mientras que en la dermatitis herpetiforme se habla de anticuerpos IgA específicos para la eTG y anticuerpos reactivos IgA dirigidos contra tTG y eTG. Como resultado de esta respuesta inmune contra la eTG, se desarrolla un clivaje subepidérmico de la lámina lúcida, lo que coincide con los depósitos de inmunoglobulina $A$ y complemento en las papilas dérmicas [24,25].

Se estima que alrededor de un $5 \%$ de los pacientes con EC pueden desarrollar una dermatitis herpetiforme a lo largo de su vida, a cualquier edad y con mayor prevalencia en pacientes del sexo masculino. El tratamiento que debe ser instaurado es la DLG [24].

\section{Manifestaciones neurológicas}

Aunque los síntomas nerviosos no son la manifestación extraintestinal más común en $E C$, es un escenario al que se enfrentan pacientes y profesionales de la salud con cierta frecuencia $[26,27]$. El origen de la presentación de síntomas neurológicos secundarios a la EC puede ser clasificado en dos vías: 1) la deficiencia de nutrientes por el fenómeno de malabsorción, y, 2) la desregulación en procesos inflamatorios asociada a la naturaleza autoinmune de la enfermedad. 
Como se ha expuesto anteriormente, las deficiencias nutricionales son una consecuencia importante de la EC. Se ha demostrado que pacientes con EC, aun sin síntomas intestinales evidentes, presentan deficiencias significativas en los niveles de zinc, albúmina, cobre, vitamina B12 y folato, respecto a controles sanos [28]. La deficiencia de micronutrientes producto de la malabsorción que se presenta en pacientes de EC puede provocar la aparición de síndromes neurológicos.

La literatura científica y la práctica clínica respaldan ampliamente la deficiencia de vitaminas ( $D, E$ y complejo $B$ ) y minerales (cobre y zinc) como causa de trastornos nerviosos centrales y periféricos, incluyendo neuropatías, parestesias, ataxias, delirio, demencia y depresión, entre otros [29]. De hecho, se han reportado casos de neuropatías en pacientes con EC cuya causa se atribuye a la deficiencia de micronutrientes como el cobre y la vitamina $E$, producto de la malabsorción [30,31].

Agravando este escenario, la preocupación por las deficiencias nutricionales en pacientes de EC no se debe únicamente al fenómeno de malabsorción en pacientes no controlados, sino que también se ha reportado un desbalance en la ingesta de nutrientes como el folato, magnesio y zinc en pacientes pediátricos, producto del seguimiento de una DLG [32], una de las estrategias terapéuticas más utilizadas en el manejo de la EC.

Como se mencionó anteriormente, otra de las rutas etiológicas para los trastornos nerviosos secundarios a EC es la desregulación de procesos inflamatorios en el marco de una enfermedad autoinmune. Para esta perspectiva, la evidencia en la literatura resulta más controversial y la descripción mecanís- tica no ha sido completamente dilucidada aún.

En este sentido, un estudio prospectivo con pacientes recién diagnosticados con EC plantea la asociación de síntomas neurológicos como ataxias, neuropatías, cefalea e inestabilidad en la marcha con la presencia de anticuerpos anti-transglutaminasa 6 (TG6) en el suero. Además, los mismos autores reportan que los pacientes seropositivos para anti-TG6, presentan atrofia de regiones cerebrales subcorticales en proporciones significativamente mayores a los sujetos sin autoanticuerpos TG6 [33].

La TG6, también conocida como transglutaminasa neuronal, se expresa predominantemente en un subconjunto de neuronas con un importante rol en la neurogénesis. Al igual que la tTG o TG2, la TG6 puede desamidar, transamidar y formar complejos de hapteno con el gluten, aunque en menor medida [34]. Por lo tanto, si se da una disrupción de las barreras hematoencefálica o hematonerviosa, la TG6 puede conjugarse con antígenos derivados del gluten y, a partir de ahí, desencadenar una cascada de señalización desde los linfocitos T CD4+ que reconocen el gluten, hacia linfocitos B específicos de TG6, lo que lleva a la producción de autoanticuerpos anti-TG6 de manera similar a los anticuerpos anti-TG2 [35].

También se ha hecho referencia al hallazgo de anticuerpos anti-gangliósidos y autoanticuerpos contra otros antígenos neuronales, y su posible asociación con manifestaciones neurológicas. Se ha hipotetizado que la interacción gangliósido-gliadina y la posible formación de haptenos pueden desencadenar una respuesta autoinmune a los gangliósidos en pacientes con EC 
[36]. De hecho, se ha encontrado una asociación significativa entre la presencia de anticuerpos anti-gangliósidos y las manifestaciones neurológicas en pacientes con EC [37].

Asimismo, se ha identificado la reactividad cruzada de los anticuerpos antigliadina contra la proteína sinapsina I. Se ha postulado que tal reactividad cruzada podría orientar sobre el mecanismo patogénico de las manifestaciones neurológicas en pacientes con EC [38].

Desde un punto de vista contrario, Ferlazzo y colaboradores afirman que los anticuerpos anti-TG6 no pueden considerarse un biomarcador diagnóstico para otras complicaciones neurológicas de la EC, al no mostrar asociación con las convulsiones relacionadas con calcificaciones cerebrales, además de que la patogénesis de estas afecciones aún no se ha dilucidado [39]. Aún más, otros estudios no han podido demostrar la asociación entre los anticuerpos anti-gangliósidos y las manifestaciones neurológicas en pacientes de EC [40].

Ante esta disyuntiva en cuanto a los marcadores serológicos, la recomendación más conveniente sería no dejarlos de lado y utilizarlos con cautela, siempre buscando una correlación con el cuadro clínico o factores de riesgo en la historia clínica del paciente con EC.

\section{Otras condiciones relacionadas con EC}

Cabe mencionar que, además de las patologías previamente mencionadas, existen otras condiciones que pueden resultar de la enfermedad celíaca; tal es el caso de las enfermedades tiroi- deas autoinmunes, la diabetes mellitus y la infertilidad.

En el caso de los trastornos tiroideos autoinmunes, entre $4,1 \%$ a $9,3 \%$ de los pacientes con EC presentan enfermedades tiroideas de origen autoinmune. La asociación con EC resulta de varios factores de riesgo compartidos como son las alteraciones en niveles de interleuquinas, pertenecer al sexo femenino, ciertos subtipos de HLA clase II y la herencia poligénica. Además, se ha descrito que en pacientes celíacos, la mala absorción de selenio, yodo y vitamina $D$ actúan como factores predisponentes a lesión de la glándula tiroidea [41].

Con mayor relevancia en los últimos años, se ha estudiado la relación existente entre los mecanismos fisiopatológicos de la EC y la infertilidad, donde se ha determinado que procesos autoinmunes específicos $\mathrm{y} / \mathrm{o}$ deficiencia nutricional al momento o previo a la concepción, pueden conducir a deficiencia ovárica. Por ejemplo, la disminución en el consumo de nutrientes como el zinc y el selenio, es capaz de provocar alteración de la síntesis y secreción de hormonas como la luteinizante (LH) y folículo estimulante (FSH), induciendo un eje hipófisis-ovárico anormal, preeclampsia y abortos espontáneos [42].

Respecto a la diabetes mellitus tipo 1, existe una asociación principalmente de prevalencia entre esta y la EC, donde se estima que al menos el $4 \%$ de los pacientes con diabetes mellitus tipo 1 también cursan con enfermedad celíaca. Esta asociación se encuentra explicada desde la susceptibilidad genética, ya que el HLA DR3-DQ2 y el DR4-DQ8 son compartidos por ambas enfermedades [43]. 


\section{Apoyo del laboratorio clínico en el seguimiento de complicaciones de la EC}

Se han revisado hasta el momento las principales complicaciones o patologías secundarias a la EC, sus orígenes e implicaciones. En este punto resulta importante realizar un aporte en cuanto a los análisis de laboratorio que pueden ser de utilidad en el seguimiento de los pacientes con EC.

En este sentido, se busca una detección temprana mediante el diagnóstico oportuno de pacientes con síntomas compatibles con ECo, incluso, una detección anticipada utilizando tamizajes masivos, todo ello con el fin de iniciar un manejo adecuado (como una DLG, por ejemplo) desde etapas tempranas de la enfermedad (prevención secundaria) [44]. También, desde un enfoque de prevención terciaria (mitigación de los daños a la salud mediante un adecuado monitoreo y un tratamiento oportuno), ciertos análisis de laboratorio permiten evaluar la aparición y/o severidad de complicaciones como las anteriormente mencionadas [44]. Un conjunto de pruebas de laboratorio para dicho fin se resume en la tabla 1.

Respecto a las pruebas de laboratorio relacionadas con el monitoreo de (micro) nutrientes, es importante realizar la siguiente aclaración: aunque la mayoría de deficiencias nutricionales o de micronutrientes puede ocurrir producto de una malabsorción en pacientes con EC no controlados, el monitoreo de estos parámetros puede ser importante incluso en aquellos pacientes sin síntomas que llevan una DLG. Esto debido a que se ha reportado que la calidad nutricional de una cantidad considerable de productos libres de gluten no es equiparable a sus homólogos con gluten $[55,56]$.
De todo lo anterior se desprende el papel clave que tiene el laboratorio clínico en el seguimiento y preservación de la calidad de vida de los pacientes con $E C$, al igual que otros servicios de apoyo diagnóstico radiológicos, neurológicos e histopatológicos, cuyos importantes aportes escapan del enfoque de la presente revisión.

\section{Conclusiones}

La enfermedad celíaca comprende una amplia gama de manifestaciones clínicas que van desde la parte intestinal hasta formas no clásicas que no involucran el sistema digestivo, lo que permite evidenciar cómo el componente autoinmune de carácter sistémico es responsable del desarrollo de múltiples patologías secundarias.

Conviene mencionar que muchas de las complicaciones que resultan de la enfermedad celíaca y que culminan en el desarrollo de patologías secundarias, tienen su origen en el fenómeno de malabsorción por la atrofia de las vellosidades intestinales, tales como las manifestaciones neurológicas, la anemia y la disminución de la densidad ósea, por lo que es necesario el monitoreo de estos pacientes para evitar deficiencia de nutrientes.

Dado el aumento de la prevalencia de pacientes con EC, sería adecuado una correcta valoración clínica con el fin de confirmar o descartar el padecimiento, ya que muchos de los afectados cursan asintomáticos para la enfermedad, pero desarrollan otras manifestaciones o patologías secundarias que se pueden evitar mediante un manejo integral de los hallazgos clínicos y de laboratorio, con el fin de evitar futuras complicaciones. 
Tabla 1. Pruebas de laboratorio relevantes en la detección y seguimiento de condiciones secundarias a la enfermedad celíaca

\begin{tabular}{lll}
\hline Prueba de laboratorio & Utilidad & $\begin{array}{l}\text { Patología } \\
\text { secundaria } \\
\text { a la que orienta }\end{array}$ \\
\hline
\end{tabular}

Alanino aminotransferasa

(ALT)

Aspartato aminotransferasa

(AST)

Anticuerpos anti-

gangliósidos

(lgG/lgM anti-GM1, anti-

GD1a, anti-GD1b, anti-GQ1b)

Anticuerpos anti-

transglutaminasa neuronal

(lgG/lgM anti-TG6)

Anticuerpos anti-

transglutaminasa epidérmica

(Ig A anti-eTG, IgA anti-TG3)

\section{Vitamina B3 \\ (niacina; medida como \\ nicotinamida, ácido \\ nicotínico en sangre o N1- \\ metilnicotinamida en orina)}

Vitamina B6 (piridoxina o piridoxal fosfato)

\section{Vitamina B9 \\ (ácido fólico o folato)}

Marcador de daño hepático

Daño hepático

[16-18]

Marcador de daño hepático Daño hepático

$[16,18]$

Candidato a marcador de procesos de autoinmunidad en los sistemas nervioso central y periférico, aunque su especificidad es debatida

Candidato a marcador de procesos de autoinmunidad en los sistemas nervioso central y periférico, aunque su especificidad es debatida

Manifestaciones neurológicas

Marcador serológico sensible para la confirmación de una etiología autoinmune en lesiones cutáneas

Monitoreo de los niveles de esta vitamina para evitar manifestaciones neurológicas producto de su deficiencia

Monitoreo de los niveles para evitar manifestaciones neurológicas producto de su deficiencia

Monitoreo de los niveles para evitar manifestaciones hematológicas o

Anemia

Manifestaciones neurológicas

neurológicas producto de su deficiencia

Monitoreo de los niveles para evitar manifestaciones

Vitamina B12 (cobalamina) hematológicas o neurológicas producto de su deficiencia

Manifestaciones neurológicas

Manifestaciones neurológicas

Monitoreo de posible deficiencia producto de la malabsorción del mineral, lo cual puede incidir en el balance metabólico óseo

Monitoreo de los niveles

Vitamina $\mathrm{E}$ (tocoferol o $\alpha$-tocoferol)
Anemia, manifestaciones neurológicas

Osteoporosis

Manifestaciones neurológicas para evitar manifestaciones neurológicas producto de su deficiencia 


\begin{tabular}{|c|c|c|c|}
\hline Prueba de laboratorio & Utilidad & $\begin{array}{l}\text { Patología } \\
\text { secundaria } \\
\text { a la que orienta }\end{array}$ & Referencias \\
\hline Calcio sérico & $\begin{array}{l}\text { Monitoreo de hipocalcemia } \\
\text { producto de la malabsorción } \\
\text { del mineral, lo cual puede } \\
\text { incidir en el balance } \\
\text { metabólico óseo }\end{array}$ & Osteoporosis & {$[15,47]$} \\
\hline Cobre sérico & $\begin{array}{l}\text { Monitoreo de los niveles } \\
\text { como micronutriente para } \\
\text { evitar manifestaciones } \\
\text { neurológicas producto de su } \\
\text { deficiencia }\end{array}$ & $\begin{array}{l}\text { Manifestaciones } \\
\text { neurológicas }\end{array}$ & {$[30,31]$} \\
\hline Ferritina & $\begin{array}{l}\text { Marcador sanguíneo } \\
\text { sensible a las disminuciones } \\
\text { en las reservas de hierro, } \\
\text { presentando alteraciones } \\
\text { incluso antes del desarrollo } \\
\text { de anemia }\end{array}$ & Anemia & {$[21,23,48]$} \\
\hline Hemograma completo & $\begin{array}{l}\text { Estudio de los índices } \\
\text { hemáticos y hallazgos } \\
\text { morfológicos que permiten } \\
\text { determinar si hay anemia } \\
\text { y orientar sobre su posible } \\
\text { etiología }\end{array}$ & Anemia & {$[21,23]$} \\
\hline Perfil del hierro & $\begin{array}{l}\text { Medición del hierro sérico, } \\
\text { la capacidad total de fijación } \\
\text { del hierro y el porcentaje de } \\
\text { saturación de transferrina. } \\
\text { Todo ello con el fin de } \\
\text { determinar si existe una } \\
\text { deficiencia de hierro }\end{array}$ & Anemia & {$[21,23,48]$} \\
\hline Fosfatasa alcalina & $\begin{array}{l}\text { Marcador relacionado con el } \\
\text { estado del hígado y con el } \\
\text { balance metabólico óseo }\end{array}$ & $\begin{array}{l}\text { Osteoporosis, } \\
\text { daño hepático }\end{array}$ & {$[49,50]$} \\
\hline Fosfato sérico & $\begin{array}{l}\text { Monitoreo de los } \\
\text { biomarcadores involucrados } \\
\text { en el balance metabólico } \\
\text { óseo }\end{array}$ & Osteoporosis & {$[49,50]$} \\
\hline $\begin{array}{l}\text { Hormona paratiroidea } \\
\text { (PTH) }\end{array}$ & $\begin{array}{l}\text { Monitoreo de los } \\
\text { biomarcadores involucrados } \\
\text { en el balance metabólico } \\
\text { óseo. En este caso, } \\
\text { para la detección de } \\
\text { hiperparatiroidismo } \\
\text { secundario por } \\
\text { hipocalcemia. Además, } \\
\text { se ha evidenciado un } \\
\text { mayor riesgo de trastornos } \\
\text { paratiroideos primarios en } \\
\text { pacientes con EC }\end{array}$ & Osteoporosis & {$[51,52]$} \\
\hline
\end{tabular}




\begin{tabular}{|c|c|c|c|}
\hline Prueba de laboratorio & Utilidad & $\begin{array}{l}\text { Patología } \\
\text { secundaria } \\
\text { a la que orienta }\end{array}$ & Referencias \\
\hline $\begin{array}{l}\text { Propéptido amino-terminal } \\
\text { del procolágeno tipo } 1 \\
(\mathrm{P} 1 \mathrm{NP}) \text {, amino-telopéptido } \\
\text { reticulado de colágeno } \\
\text { tipo I en orina (NTx), } \\
\text { deoxipiridinolina (pyrilinks D) }\end{array}$ & $\begin{array}{l}\text { Marcadores para el } \\
\text { monitoreo de la resorción } \\
\text { ósea. Su aplicabilidad se } \\
\text { recomienda principalmente } \\
\text { en investigación }\end{array}$ & Osteoporosis & [53] \\
\hline Zinc sérico & $\begin{array}{l}\text { Monitoreo de los niveles de } \\
\text { zinc como micronutriente } \\
\text { para evitar manifestaciones } \\
\text { neurológicas producto de su } \\
\text { deficiencia }\end{array}$ & $\begin{array}{l}\text { Manifestaciones } \\
\text { neurológicas }\end{array}$ & {$[28,54]$} \\
\hline
\end{tabular}

\section{Referencias}

1. Organización Mundial de la Salud. Clasificación internacional de enfermedades para estadísticas de mortalidad y morbilidad. 11a revisión. DA 95-Enfermedad celíaca. Ginebra: OMS; 2019. Acceso 15 de mayo de 2020. Disponible en https://icd.who.int/browse11/l-m/ es\#/http://id.who.int/icd/entity/2005943638.

2. Salazar C, García-Cárdenas JM, Paz y-Miño

C. Understanding celiac disease from genetics to the future diagnostic strategies. Clin Med Insights Gastroenterol 2017;10: 80502508. https://doi.org/10.1177/1179552217712249.

3. Dieli-Crimi R, Cénit MC, Núñez C. The genetics of celiac disease: A comprehensive review of clinical implications. J Autoimmun 2015;64:26-41. https://doi.org/https://doi. org/10.1016/j.jaut.2015.07.003.

4. Parzanese I, Qehajaj D, Patrinicola F, Aralica M, Chiriva-Internati M, Stifter S, et al. Celiac disease: From pathophysiology to treatment. World J Gastrointest Pathophysiol 2017;8:2738. https://doi.org/10.4291/wjgp.v8.i2.27.

5. Singh $P$, Arora A, Strand TA, Leffler DA, Catassi C, Green PH, et al. Global prevalence of celiac disease: Systematic review and meta-analysis. Clin Gastroenterol Hepatol 2018;16:823-836.e822. https://doi.org/10.1016/j.cgh.2017.06.037.

6. West J, Fleming KM, Tata LJ, Card TR, Crooks CJ. Incidence and prevalence of celiac disease and dermatitis herpetiformis in the UK over two decades: population-based study. Am J
Gastroenterol 2014;109:757-768. https://doi. org/10.1038/ajg.2014.55.

7. Altobelli E, Paduano R, Petrocelli R, Di Orio F. Burden of celiac disease in Europe: a review of its childhood and adulthood prevalence and incidence as of September 2014. Ann lg 2014;26:485-498. https://doi.org/10.7416/ ai.2014.2007.

8. Lerner A, Lopez F, Schmiedl A, Matthias T. The underdiagnosed enemy: Africa goes celiac? Int J Celiac Dis 2019;7:9-12.

9. Jabeen. Celiac disease. From the bench to the clinic. In: Rodrigo L, Hernández-Lahoz C, eds. Complications of Celiac Disease. Londres: IntechOpen; 2019. p. 20. https://doi.org/https:// doi.org/10.5772/intechopen.80465.

10. Stier K, Lewis SK, Bhagat G, Green PHR. Research publication trends regarding the extraintestinal manifestations of celiac disease. Inform Med Unlocked 2019;17:100242. https://doi.org/ https://doi.org/10.1016/j.imu.2019.100242.

11. Valderas JM, Starfield B, Sibbald B, Salisbury C, Roland M. Defining comorbidity: implications for understanding health and health services. Ann Fam Med 2009;7:357-363. https://doi. org/10.1370/afm.983.

12. Laurikka $\mathbf{P}$, Nurminen $S$, Kivelä $L$, Kurppa K. Extraintestinal manifestations of celiac disease: Early detection for better long-term outcomes. Nutrients 2018;10:1015. https://doi. org/10.3390/nu10081015.

13. Bartl R, Bartl C. Definition of Osteoporosis. In: Bartl R, Bartl C, eds. Bone Disorders. Suiza: Sprin- 
ger, Cham; 2016. p. 109-110. https://doi.org/ https://doi.org/10.1007/978-3-319-29182-6.

14. Brunetto OH. Osteoporosis en pediatría. Rev Argent Endocrinol Metab 2006;43:90-108.

15. Jódar-Gimeno E. Osteoporosis secundarias. Medicine 2014;11:3535-3544. https://doi. org/10.1016/S0304-5412(14)70812-1.

16. Zanini $B$, Baschè $R$, Ferraresi A, Pigozzi MG, Ricci C, Lanzarotto F, et al. Factors that contribute to hypertransaminasemia in patients with celiac disease or functional gastrointestinal syndromes. Clin Gastroenterol Hepatol 2014;12:804-810.e802. https://doi. org/10.1016/j.cgh.2013.10.033.

17. Hatanaka SA, Silva N, Dantas-Correa E, Schiavon L, Narciso-Schiavon J. The effect of a gluten-free diet on alanine aminotransferase $(\mathrm{ALT})$ in celiac patients. Rev Colomb Gastroenterol 2015;30:412-418.

18. Cantarero-Vallejo MD, Gómez-Camarero J, Menchén L, Pajares-Díaz JA, Lo lacono 0. Daño hepático y enfermedad celíaca. Rev Esp Enferm Dig 2007;99:648-652.

19. Barbero-Villares A, Moreno-Monteagudo JA, Moreno-Borque R, Moreno-Otero R. Afectación hepática en la enfermedad celíaca. Gastroenterol Hepatol 2008;31:25-28. https://doi. org/10.1157/13114567.

20. Alonso-Cotoner $C$, Casellas-Jordá F, Chicharro-Serrano ML, Torres-Ramírez Id, Malagelada-Benaprés JR. Ferropenia: no siempre son pérdidas. An Med Interna 2003;20:11-15.

21. Mearin $F$, Balboa $A$, Castells $A$, Domínguez JE, Esteve M, García-Erce JA, et al. Anemia ferropénica y uso de hierro endovenoso en patología digestiva. Gastroenterol Hepatol 2010;33:605-613. https://doi.org/10.1016/j.gastrohep.2010.08.001.

22. Kumar V, Parmeshwarappa S, Vadde A. Celiac disease manifesting as isolated cobalamin deficiency megaloblastic anemia: Case series and review. J Sci Soc 2014;41:211-214. https:// doi.org/10.4103/0974-5009.141246.

23. Berry N, Basha J, Varma N, Varma S, Prasad KK, Vaiphei K, et al. Anemia in celiac disease is multifactorial in etiology: A prospective study from India. JGH Open 2018;2:196-200. https:// doi.org/10.1002/jgh3.12073.
24. Sanjinés L, Martínez M, Magliano J. Dermatitis herpetiforme como carta de presentación de la enfermedad celíaca. Rev Urug Med Int 2016;1:3.

25. Fonseca E. Manifestaciones cutáneas en la enfermedad celiaca. Pediatrika 2003;23:38-39.

26. Işıkay S, Kocamaz H, Sezer S, Özkars MY, Işıkay N, Filik B, et al. The frequency of epileptiform discharges in celiac disease. Pediatr Neurol 2015;53:78-82. https://doi.org/10.1016/j. pediatrneurol.2015.02.006.

27. Croall ID, Sanders DS, Hadjivassiliou M, Hoggard N. Cognitive deficit and white matter changes in persons with celiac disease: A population-based study. Gastroenterology 2020;158:2112-2122. https://doi. org/10.1053/j.gastro.2020.02.028.

28. Bledsoe AC, King KS, Larson JJ, Snyder M, Absah I, Choung RS, et al. Micronutrient deficiencies are common in contemporary celiac disease despite lack of overt malabsorption symptoms. Mayo Clin Proc 2019;94:1253-1260. https://doi. org/10.1016/j.mayocp.2018.11.036.

29. Maxwell PJ, Montgomery SC, Cavallazzi R, Martindale RG. What micronutrient deficiencies should be considered in distinct neurological disorders? Curr Gastroenterol Rep 2013;15:331. https://doi.org/10.1007/s11894013-0331-7.

30. Henri-Bhargava A, Melmed C, Glikstein R, Schipper HM. Neurologic impairment due to vitamin e and copper deficiencies in celiac disease. Neurology 2008;71:860-861. https://doi. org/10.1212/01.wnl.0000325473.13088.18.

31. Avila JD, Lacomis D. Proximal limb weakness in a patient with celiac disease: Copper deficiency, gluten sensitivity, or both as the underlying cause? Case Rep Neurol Med 2016;2016:5415949. https://doi.org/10.1155/2016/5415949.

32. Di Nardo G, Villa MP, Conti L, Ranucci G, Pacchiarotti C, Principessa L, et al. Nutritional deficiencies in children with celiac disease resulting from a gluten-free diet: A systematic review. Nutrients 2019;11:1588. https://doi. org/10.3390/nu11071588.

33. Hadjivassiliou M, Croall ID, Zis $P$, Sarrigiannis PG, Sanders DS, Aeschlimann P, et al. Neurologic deficits in patients with newly diagnosed 
celiac disease are frequent and linked with autoimmunity to transglutaminase 6. J Clin Gastroenterol Hepatol 2019;17:2678-2686.e2672. https://doi.org/10.1016/j.cgh.2019.03.014.

34. Stamnaes J, Dorum S, Fleckenstein B, Aeschlimann D, Sollid LM. Gluten T cell epitope targeting by TG3 and TG6; implications for dermatitis herpetiformis and gluten ataxia. Amino Acids 2010;39:1183-1191. https://doi. org/10.1007/s00726-010-0554-y.

35. Yu XB, Uhde M, Green PH, Alaedini A. Autoantibodies in the extraintestinal manifestations of celiac disease. Nutrients 2018;10:1123. https://doi.org/10.3390/nu10081123.

36. Alaedini A, Latov N. Transglutaminase-independent binding of gliadin to intestinal brush border membrane and GM1 ganglioside. J Neuroimmunol 2006;177:167-172. https://doi. org/10.1016/j.jneuroim.2006.04.022.

37. Volta U, De Giorgio R, Granito A, Stanghellini V, Barbara G, Avoni P, et al. Anti-ganglioside antibodies in coeliac disease with neurological disorders. Dig Liver Dis 2006;38:183-187. https://doi.org/10.1016/j.dld.2005.11.013.

38. Alaedini A, Okamoto $H$, Briani $C$, Wollenberg K, Shill HA, Bushara KO, et al. Immune crossreactivity in celiac disease: anti-gliadin antibodies bind to neuronal synapsin I. J Immunol 2007;178:6590-6595. https://doi.org/10.4049/ jimmunol.178.10.6590.

39. Ferlazzo E, Polidoro S, Gobbi G, Gasparini S, Sueri C, Cianci V, et al. Epilepsy, cerebral calcifications, and gluten-related disorders: Are anti-transglutaminase 6 antibodies the missing link? Seizure 2019;73:17-20. https://doi. org/10.1016/j.seizure.2019.10.012.

40. Briani C, Zara G, Alaedini A, Grassivaro F, Ruggero S, Toffanin E, et al. Neurological complications of celiac disease and autoimmune mechanisms: a prospective study. J Neuroimmunol 2008;195:171-175. https://doi. org/10.1016/j.jneuroim.2008.01.008.

41. Real RE, Valenzuela JA, González NR. Enfermedad celiaca silente en pacientes adultos con enfermedades tiroideas autoinmunes. An Fac Cienc Méd 2020;53:71-80. https:// doi.org/http://dx.doi.org/10.18004/anales/2020.053.01.71-080.
42. Navarro G. Enfermedad celiaca e infertilidad no explicada: papel del tamizaje. Revisión de literatura. Rev Cien Sal Int Con 2020;4:115-122 https:// doi.org/10.34192/cienciaysalud.v4i3.153.

43. Ramírez-Benítez Al, Miranda-Ojeda MC, Ferreira L, Palacios-Lugo MC, Jiménez-González JT. Enfermedad celiaca y diabetes mellitus tipo 1: asociación y características clínicas. Rev virtual Soc Parag Med Int 2014;1:8-17.

44. Mearin ML. The prevention of coeliac disease. Best Pract Res Clin Gastroenterol 2015;29:493 501. https://doi.org/10.1016/j.bpg.2015.04.003.

45. Rose C, Armbruster FP, Ruppert J, Igl BW, ZiIlikens D, Shimanovich I. Autoantibodies against epidermal transglutaminase are a sensitive diagnostic marker in patients with dermatitis herpetiformis on a normal or gluten-free diet. J Am Acad Dermatol 2009;61:39-43. https://doi. org/10.1016/j.jaad.2008.12.037.

46. Borroni G, Biagi F, Ciocca O, Vassallo C, Carugno A, Cananzi $\mathbf{R}$, et al. IgA anti-epidermal transglutaminase autoantibodies: a sensible and sensitive marker for diagnosis of dermatitis herpetiformis in adult patients. J Eur Acad Dermatol Venereol 2013;27:836-841. https://doi. org/10.1111/j.1468-3083.2012.04586.x.

47. Larussa T, Suraci E, Nazionale I, Abenavoli L, Imeneo M, Luzza F. Bone mineralization in celiac disease. Gastroenterol Res Pract 2012;2012:198025. https://doi. org/10.1155/2012/198025.

48. Powers JM, O'Brien SH. How I approach iron deficiency with and without anemia. Pediatr Blood Cancer 2019;66:e27544. https://doi. org/10.1002/pbc.27544.

49. Heaney RP. Phosphorus nutrition and the treatment of osteoporosis. Mayo Clin Proc 2004;79:91-97. https://doi.org/10.4065/79.1.91.

50. Saadati N, Khodashahi M, Naghibzadeh B, Adibi E. Osteomalacia with looser zones caused by celiac disease. Rheumatol Res 2017;2:109-112. https://doi.org/10.22631/rr.2017.69997.1027.

51. Patel SR, Shashaty RJ, Denoux P. Nutritional nightmare: Hypoparathyroidism secondary to celiac disease. Am J Med 2017;130:e525. https://doi.org/10.1016/j.amjmed.2017.07.032.

52. Ludvigsson JF, Kämpe O, Lebwohl B, Green PH, Silverberg SJ, Ekbom A. Primary hyperpa- 
rathyroidism and celiac disease: a populationbased cohort study. J Clin Endocrinol Metab 2012;97:897-904. https://doi.org/10.1210/ jc.2011-2639.

53. Fouda MA, Khan AA, Sultan MS, Rios LP, McAssey K, Armstrong D. Evaluation and management of skeletal health in celiac disease: position statement. Can J Gastroenterol 2012;26:819829. https://doi.org/10.1155/2012/823648.

54. Wierdsma NJ, van Bokhorst-de van der Schueren MAE, Berkenpas M, Mulder CJJ, van Bodegraven AA. Vitamin and mineral deficiencies are highly prevalent in newly diagnosed celiac disease patients. Nutrients 2013;5:39753992. https://doi.org/10.3390/nu5103975.

55. Elliott C. The nutritional quality of gluten-free products for children. Pediatrics 2018;142:e20180525. https://doi.org/10.1542/ peds.2018-0525.

56. Larretxi I, Txurruka I, Navarro V, Lasa A, Bustamante M, Fernández-Gil MDP, et al. Micronutrient analysis of gluten-free products: Their low content is not involved in gluten-free diet imbalance in a cohort of celiac children and adolescent. Foods 2019;8:321. https://doi. org/10.3390/foods 8080321 . 